\title{
Exploration of Reduced Doses and Short-Cycle Therapy for Darunavir/ Cobicistat in Patients with HIV Using Population Pharmacokinetic Modeling and Simulations
}

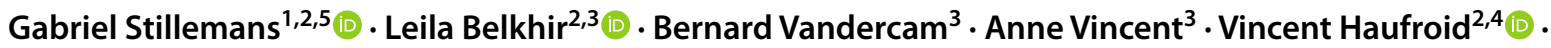 \\ Laure Elens $\mathbf{s}^{1,2} \mathbb{0}$
}

Published online: 22 July 2020

(c) The Author(s) 2020

\begin{abstract}
Background and objectives Protease inhibitors such as darunavir are an important therapeutic option in the anti-human immunodeficiency virus arsenal. Current dosage guidelines recommend using cobicistat- or ritonavir-boosted darunavir $800 \mathrm{mg}$ every $24 \mathrm{~h}$ (q24h) in protease inhibitor-naïve patients, or ritonavir-boosted darunavir $600 \mathrm{mg}$ q12h in experienced patients. However, darunavir displays a large, poorly characterized, inter-individual pharmacokinetic variability. The objectives of this study were to investigate the pharmacokinetics of darunavir and to elucidate the sources of its inter-individual variability using population pharmacokinetic modeling. Then, to determine the appropriateness of current treatment guidelines and the feasibility of alternative dosing regimens in a representative cohort of adult patients using simulations.

Methods Sparse pharmacokinetic samples were collected in 127 patients with human immunodeficiency virus type 1 infection, then supplemented with rich sampling data from a subset of 12 individuals. Data were analyzed using the nonlinear mixed-effects modeling software NONMEM. The effect of reduced doses (600 mg q24h and $400 \mathrm{mg}$ q24h) or reduced frequency of administration ( $800 \mathrm{mg}$ q24h for 5 days followed by 2 days of treatment interruption) was simulated.

Results Our model adequately described the pharmacokinetics of darunavir. Predictors of individual exposure were CYP3A5*3 and SLCO3A1 rs8027174 genotypes, sex, and alpha-1 acid glycoprotein level. No relationship was apparent between darunavir area under the curve and treatment efficacy or safety. For reduced dose regimens, darunavir concentrations remained above the protein binding-corrected $\mathrm{EC}_{50}$ in the majority of subjects. More stringent pharmacokinetic targets were not reached in a significant proportion of patients.

Conclusions These results add to the growing body of evidence that darunavir-based therapy could be simplified to reduce costs and toxicity, as well as to improve patient compliance. However, the heterogeneity in pharmacokinetic response should be considered when assessing whether individual patients could benefit from a particular regimen, for instance through the use of population pharmacokinetic models.
\end{abstract}

Clinical Trial Registration ClinicalTrials.gov identifier: NCT03101644, date of registration: 5 April, 2017.

\section{Introduction}

According to international guidelines, protease inhibitors (PIs), in combination with a background regimen of two nucleoside reverse transcriptase inhibitors (NRTI), are a

Electronic supplementary material The online version of this article (https://doi.org/10.1007/s40262-020-00920-z) contains supplementary material, which is available to authorized users.

Gabriel Stillemans

gabriel.stillemans@uclouvain.be

Extended author information available on the last page of the article first-line option for the management of human immunodeficiency virus type 1 (HIV-1) infection [1,2]. Darunavir (DRV) is the preferred PI, but must be coadministered with a pharmacokinetic (PK) booster, either ritonavir (RTV) or cobicistat (COB), to maximize exposure. Standard dosages include DRV/RTV 800/100 mg every $24 \mathrm{~h}$ (q24h) or DRV/ COB 800/150 mg q24h for PI-naïve patients [3] (or those pretreated with PIs but with no known resistance mutations) [4], and DRV/RTV 600/100 mg every $12 \mathrm{~h}$ (q12h) for patients presenting with PI resistance mutations [5-8]. One of the main advantages of DRV is its high genetic barrier to resistance as it binds to a highly conserved domain of the viral protease [9-11]. This makes it useful in patients with 


\section{Key Points}

A population pharmacokinetic model of darunavir was developed and validated. CYP3A5 and SLCO3A1 genotypes, alpha-acid glycoprotein level, and sex were predictors of darunavir pharmacokinetics.

Reduced dosages (600 mg every $24 \mathrm{~h}$ and $400 \mathrm{mg}$ every $24 \mathrm{~h}$ ) were predicted to be safe in the majority of patients. Our model could be used to assess changes in exposure for various alternative regimens in specific individuals.

incomplete adherence because subtherapeutic exposure is unlikely to result in the emergence of resistance mutations, making re-challenge with the same drug possible.

However, treatment with DRV is rendered delicate by its complex spectrum of drug-drug interactions (DDIs). Not only must certain drugs be avoided to ensure adequate exposure to antiretrovirals (ARVs), but DRV and its boosters can also inhibit (and sometimes induce) various cytochromes and transporters, thereby affecting other therapies [12-15]. Indeed, DRV is a substrate for cytochrome P450 (CYP) $3 \mathrm{~A}$; a substrate for efflux transports of the $\mathrm{ABC}$ family ABCB1 (P-glycoprotein) and ABCC2; a substrate for influx transporters of the OATP family OATP1A2, OATP1B1, and OATP1B3; and has the potential to inhibit CYP3A4, OATP1B1, and OATP1B3. Meanwhile, RTV and COB exert slightly different effects: both are capable of inhibiting CYP3A, OATP1B1, and OATP1B3. Ritonavir may also induce other CYP isoforms and pregnane $\mathrm{X}$ receptors, while COB is more selective in this regard [16].

Darunavir pharmacokinetics are highly variable between individuals, although the sources of this heterogeneity remain poorly understood. Single nucleotide polymorphisms (SNPs) in genes coding for biotransformation enzymes and transporters, DDIs, and other patient-specific covariates may all contribute to this phenomenon [17]. Previous studies have already highlighted the role played by certain individual factors such as alpha-glycoprotein level [18, 19], sex [20], weight [21], age [20], SNPs in SLCO3A1 [19], and RTV exposure [21-23]. Our first aim was to use population pharmacokinetics to model this variability in a representative cohort of adult patients with HIV and to identify the best set of predictors. In addition, we evaluated whether changes in DRV pharmacokinetics were correlated with biomarkers of treatment efficacy (viral load and lymphocyte count) and toxicity (occurrence of adverse events).

Our second aim was to simulate the effect of alternative dose regimens. Simplified antiretroviral therapies (ARTs) involving fewer drugs or lower doses have gained attention as they could help to reduce toxicity, reduce costs (therefore increasing access to the drug in developing countries), and improve convenience and patient compliance. A reduction in the daily dose of DRV/RTV from 800/100 to 600/100 mg $\mathrm{q} 24 \mathrm{~h}$ was demonstrated to be safe in virologically suppressed patients [24, 25], as was a further reduction to $400 / 100 \mathrm{mg}$ q24h [26, 27]. Additionally, DRV $400 \mathrm{mg}$ plus a NRTI backbone was found to be non-inferior to standard dose lopinavir plus a NRTI backbone [27]. Ritonavir- or COB-boosted DRV monotherapy has also seen some success [28-34], even with the lower 600-mg q24h DRV dosage [35]. Although this type of regimen may expose patients to a higher risk of viral blips, they may easily be re-suppressed by restoring their initial treatment due to the aforementioned pharmacological properties of DRV. Short-cycle therapy (SCT) is a different approach consisting of alternating on- and offtreatment phases for a few days each, which would significantly reduce the drug burden for the patient. Moreover, a lower pill burden has been correlated with better adherence in patients with HIV, which could help with long-term viral suppression [36]. Based on what few data are available, SCT for DRV-containing triple combination ART appears to be a viable option, despite low plasma concentrations of DRV during the off-treatment phase [37]. The effects of both reduced doses and SCT were investigated in the present study using simulations, based on the population PK model we developed.

\section{Materials and Methods}

\subsection{Study Design}

This was a prospective study conducted at the Cliniques Universitaires Saint-Luc (Brussels, Belgium). Blood samples were obtained during routine hospital visits, once every 3-6 months on average. One sample for the quantification of ARVs was drawn in a heparinized tube at each patient visit (dataset A). Self-reported drug intake time as well as blood sampling time were recorded. One additional sample for DNA extraction was drawn in an EDTA tube at the patient's first study visit.

Intensive sampling was performed in a subset of 12 individuals selected for their high treatment compliance based on available PK data and anamnesis by the physician. In those individuals, a dose journal over the previous 4 days was requested, dose intake on the day of sampling was witnessed by a nurse, and then eight samples were collected at pre-specified time points: pre-dose, $0.5,1,2,3,4,5$, and $6 \mathrm{~h}$ post-intake (dataset B). 


\subsection{Drug Quantification}

Darunavir, RTV, and COB were quantified using a validated, ultra-performance liquid chromatography-diode array detection method [38]. Briefly, samples were spiked with the internal standard (clomipramine), buffered to alkaline $\mathrm{pH}$, then a liquid-liquid extraction was performed using methyl tert-butyl ether. Samples were centrifuged and the supernatant was evaporated to dryness by heating at $40{ }^{\circ} \mathrm{C}$ under a stream of nitrogen. The residue was reconstituted in mobile phase and $5 \mu \mathrm{L}$ was injected on an Acquity UPLC BEH C18 column. The mobile phase was a gradient of triethylammonium phosphate $(\mathrm{pH} 3)$ and acetonitrile. All reagents were of analytical grade.

The lower limit of quantification (LLOQ) was $0.1 \mathrm{mg} / \mathrm{L}$ for DRV and RTV. For COB, no LLOQ was formally defined but a threshold of $0.2 \mathrm{mg} / \mathrm{L}$ was used based on the signalto-noise ratio. The method was linear over the range of 0.1-10 mg/L for all three analytes. Samples were analyzed in batches along with external quality controls (containing DRV and RTV) and internal controls (blank plasma spiked with known amounts of all analytes, as well as previously determined patient samples). For this analytical method, our laboratory successfully participates in an external quality assessment scheme organized by the Dutch Foundation for Quality Assessment in Medical Laboratories (SKML, Nijmegen, the Netherlands); the trueness and precision of the method were within $10 \%$ for DRV and RTV. The stability of DRV in various conditions (autosampler, room temperature, freeze-thaw, and long-term storage) has been determined by other authors $[39,40]$.

\subsection{Genotyping}

DNA was isolated using the QIAamp ${ }^{\circledR}$ DNA mini-kit (Qiagen, Antwerp, Belgium) according to the manufacturer's instructions. Allelic discrimination was performed on a Step One Plus ${ }^{\mathrm{TM}}$ real-time polymerase chain reaction system using Taqman ${ }^{\circledR}$ genotyping assays (Fisher Scientific, Merelbeke, Belgium) for $A B C B 1$ rs2229109 and rs1045642; CYP3A4 rs35599367; CYP3A5 rs776746; and SLCO3A1 rs8027174 and rs4294800. These SNPs were selected based on expected clinical relevance and results from previously published studies. Haldane's exact test (as implemented in the HardyWeinberg package for R) [41] was used to check for deviation from the Hardy-Weinberg equilibrium.

\subsection{Modeling Software}

Pharmacokinetic data were analyzed using the nonlinear mixed-effects modeling software NONMEM (version 7.4.3) [42]. Additional functionality was provided by PsN (version
4.7.0) [43]. NONMEM output was processed in $\mathrm{R}$ (version 3.5.2) [44] with the npde package (version 2.0) [45].

\subsection{Structural Model}

Dataset B was analyzed on its own then pooled with dataset A. For the pooled analysis, the PRIOR routine in NONMEM was used to specify a prior distribution of all fixed and random effects (except residual variability terms) based on final estimates of the model built on dataset B [46]. The reason we thus constrained parameter estimates was because it was assumed that dataset B would be more informative, especially regarding the absorption phase. Separate structural models were first developed for DRV and COB (development of a RTV model was not possible because of a lack of data). For each drug, a one- or two-compartment disposition model was fitted to the data. Models with first-order and delayed first-order absorption were evaluated. The interaction between COB and DRV was modeled according to a linear or $\mathrm{I}_{\max }$ (Michaelis-Menten) function. To account for the nonlinear dose-exposure relationship of DRV [23, 47], dose-dependent and saturable clearance (CL) models were tested.

\subsection{Covariate Model}

The following covariates were investigated: age, body weight, race (subjects were classified as Caucasian or African, other races were treated as Caucasian), food intake with last DRV dose, plasma albumin and $\alpha 1$-acid glycoprotein (AAG), genotypes, and DDIs. Genetic covariates have already been described in Sect. 2.3. For each SNP, subjects were stratified according to their number of functional alleles, or simply as expressors vs non-expressors in the case of $C Y P 3 A 5^{*} 3$. For DDIs, concomitant drugs (ARVs and non-ARVs) were classified as CYP3A inhibitors, CYP3A inducers, or P-glycoprotein inhibitors based on published lists of interactions [48, 49]. Missing covariate values were substituted by the most recent measurement, the population median, or the most frequent category, as appropriate.

Covariates were first screened for univariate association with the empirical Bayesian estimates of PK parameters. The process of covariate stepwise selection was then partly automated using PsN, based on the decrease of the objective function value (OFV). The $\alpha$ thresholds for statistical significance were set to 0.05 for forward inclusion and 0.01 for backward elimination. The equations below were used to describe covariate relationships (for continuous covariates, linear and exponential models with normalization to the median were both considered), where cov covariate value, $\theta$ typical value, $\theta_{\text {cov }}$ covariate scale factor ( 0 for reference group), $P$ model parameter. 
Exponential : $P=\theta \times \mathrm{e}^{\theta \operatorname{cov} \times(\operatorname{cov}-\text { median })}$

Linear : $P=\theta \times\left(1+\theta_{\text {cov }} \times(\operatorname{cov}-\right.$ median $\left.)\right)$

Categorical : $P=\theta \times\left(1+\theta_{\text {cov }}\right)$

Association between model-derived area under the curve (AUC) and pharmacodynamic biomarkers was assessed using Wilcoxon tests or by calculating Spearman's rho, as appropriate. The viral load ( $>40$ or $<40$ copies $/ \mathrm{mL}$, i.e., undetectable) and CD4 + and total lymphocyte counts were used as markers of efficacy, and the occurrence of adverse events at any point during the study, stratified by type of symptom (neurological or lipodystrophy), as markers of toxicity.

\subsection{Goodness of Fit and Internal Validation}

Goodness of fit was assessed using standard graphical methods (individual and population predictions vs observations, residuals vs observations and time). Reductions in unexplained variability, shrinkage, or standard errors of estimates were additional criteria for model selection. The model was validated by verifying the normality of the normalized prediction distribution errors. Parameter uncertainty was assessed by constructing $95 \%$ confidence intervals from 1000 nonparametric bootstraps.

\subsection{Target Attainment}

Target attainment probabilities were computed by simulating DRV steady-state PK profiles based on the original dataset (1000 simulations in the absence of residual error). Evaluated strategies were DRV $800 \mathrm{mg}$ q24h, $600 \mathrm{mg}$ q24h, and $400 \mathrm{mg} \mathrm{q} 24 \mathrm{~h}$, and SCT $800 \mathrm{mg}$ q24h for 5 days. Trough concentration $\left(C_{0}\right)$ targets were set to $0.055 \mathrm{mg} / \mathrm{L}$ (protein binding-adjusted $\mathrm{EC}_{50}$ for wild-type HIV-1 protease, serving as the therapeutic target in PI-naïve patients with no resistance-associated mutations) [11], $0.55 \mathrm{mg} / \mathrm{L}$ (ten times the wild-type $\mathrm{EC}_{50}$, serving as the target in PI-experienced patients), and $2 \mathrm{mg} / \mathrm{L}$ (recommended $C_{0}$ in PI-experienced patients) [50].

\section{Results}

\subsection{Study Population}

One hundred and twenty-seven patients were enrolled, 12 of whom underwent intensive sampling. A total of 309 sparse samples (dataset A) and 96 rich samples (dataset B) were collected. For sparse samples, the post-intake time range was $1-29 \mathrm{~h}$ (median $11.9 \mathrm{~h}$ ). Concentrations were censored if the post-intake time was unknown (1.9\%), if the patient reported a lack of treatment compliance $(0.3 \%)$, or because of analytical reasons (1\%). Darunavir concentrations below the LLOQ (3.6\%) were set to half of the LLOQ.

Summary characteristics of the cohort can be found in Table 1. At baseline, median DRV treatment duration was 4.2 years, $85.8 \%$ of patients were using COB-boosted DRV, and DRV $800 \mathrm{mg}$ q24h was the most frequent dosage (91.3\%), followed by $600 \mathrm{mg} \mathrm{q} 12 \mathrm{~h}(7.9 \%)$ and $1200 \mathrm{mg}$ q24h (0.8\%). The three most common ARTs in this cohort were: DRV/tenofovir/emtricitabine (22.0\%), DRV/dolutegravir (18.1\%), and DRV/dolutegravir/lamivudine (17.3\%). Two patients (1.6\%) were using DRV $800 \mathrm{mg}$ q24h monotherapy. Frequent complaints possibly related to ART that occurred at least once during follow-up for a given subject included lipodystrophy (29.1\%) and neurological symptoms (insomnia, fatigue, or headaches) $(26.0 \%)$. Genotypes for pharmacogenes of interest are summarized in Table 2. All SNPs were in Hardy-Weinberg equilibrium with the exception of rs776746 (this deviation was no longer significant when analyzing African and Caucasian individuals separately, suggesting population stratification). Cytochrome P450 3A5 genotypes within each racial group matched their expected distribution (from, e.g., the 1000 Genomes Project) [51].

\subsection{Structural Model}

Darunavir kinetics were best described by a one-compartment model with first-order absorption and elimination. Inter-individual variability was described by an exponential error term, while a mixed (additive plus exponential) residual error was used. For dataset B only, the model fit was improved by the addition of a lag term to absorption $(\triangle \mathrm{OFV}=-36)$, and by the addition of a block diagonal matrix between random effects $(\Delta \mathrm{OFV}=-12)$. However, treating the daily dose of DRV as a covariate on CL or modeling a saturable CL did not, or prevented the model from converging. Neither the lag time nor the full random effect matrices was retained in the model developed on the combined dataset.

Similarly, COB kinetics were described by a simple onecompartment model. There appeared to be a positive correlation between DRV CL and COB CL (dataset B: rho $=0.33$, $p=0.3$, see the Electronic Supplementary Material [ESM]). Because inclusion of this relationship in a joint model on dataset B did not improve the fit, the COB portion of the model was dropped at this stage.

\subsection{Covariate Model}

For dataset B alone, two covariates were retained in the final model: all other things being equal, volume of distribution $(V)$ was decreased by $53 \%$ in subjects harboring the $C Y P 3 A 5 * 3$ allele and by $70 \%$ in Caucasian individuals 
Table 1 Cohort summary characteristics

\begin{tabular}{|c|c|c|}
\hline & $n$ & $\%$ \\
\hline Patients & 127 & \\
\hline \multicolumn{3}{|l|}{ Sampling } \\
\hline Sparse & 115 & 90.6 \\
\hline Sparse + rich & 12 & 9.4 \\
\hline \multicolumn{3}{|l|}{ DRV dose } \\
\hline $800 \mathrm{mg} \mathrm{q} 24 \mathrm{~h}$ & 116 & 91.3 \\
\hline $600 \mathrm{mg} \mathrm{q} 12 \mathrm{~h}$ & 10 & 7.9 \\
\hline $1200 \mathrm{mg} \mathrm{q} 24 \mathrm{~h}$ & 1 & 0.8 \\
\hline \multicolumn{3}{|l|}{ Booster } \\
\hline $\mathrm{COB}$ & 109 & 85.8 \\
\hline RTV & 18 & 14.2 \\
\hline \multicolumn{3}{|l|}{ Age (years) } \\
\hline Median (IQR) & $55(13)$ & \\
\hline \multicolumn{3}{|l|}{ Sex } \\
\hline Male & 85 & 66.9 \\
\hline Female & 42 & 33.1 \\
\hline \multicolumn{3}{|l|}{ Weight (kg) } \\
\hline Median (IQR) & $73(17)$ & \\
\hline \multicolumn{3}{|l|}{ Race } \\
\hline Caucasian & 67 & 52.8 \\
\hline African & 55 & 43.3 \\
\hline Other & 5 & 3.9 \\
\hline \multicolumn{3}{|l|}{ ART } \\
\hline 2 NRTIs/DRV & 29 & 22.8 \\
\hline 3TC/DTG/DRV & 23 & 18.1 \\
\hline DTG/DRV & 22 & 17.3 \\
\hline Other & 53 & 41.7 \\
\hline \multicolumn{3}{|c|}{ DRV treatment duration (years) } \\
\hline Median (IQR) & $4.21(4.82)$ & \\
\hline \multicolumn{3}{|l|}{ Viral load, RNA copies/mL } \\
\hline$<40$ & 100 & 78.7 \\
\hline$>40$ & 13 & 10.2 \\
\hline \multicolumn{3}{|l|}{ CD4 cell count $(/ \mu \mathrm{L})$} \\
\hline Median (IQR) & $564.9(348.3)$ & \\
\hline \multicolumn{3}{|l|}{ Toxicity } \\
\hline Lipodystrophy & 37 & 29.1 \\
\hline Neurological symptoms & 33 & 26.0 \\
\hline \multicolumn{3}{|l|}{ Drug-drug interactions } \\
\hline CYP3A inhibitors & 3 & 2.4 \\
\hline CYP3A inducers & 7 & 5.5 \\
\hline P-glycoprotein inhibitors & 5 & 3.9 \\
\hline
\end{tabular}

All values given at baseline (except adverse events, for which at least one occurrence during follow-up was considered)

$3 T C$ lamivudine, $A R T$ antiretroviral therapy, $C O B$ cobicistat, $C Y P$ cytochrome $\mathrm{P} 450, D R V$ darunavir, DTG dolutegravir, IQR interquartile range, NRTIS nucleoside reverse transcriptase inhibitors, $R T V$ ritonavir compared with African individuals. In the combined dataset, the CYP3A5 phenotype was associated with CL instead, with CL being $19 \%$ higher in expressors compared with non-expressors. Additionally, for the combined dataset, CL and $V$ were both decreased in subjects with higher AAG, $\mathrm{CL}$ was $21 \%$ lower in female individuals compared with male individuals, and $V$ was increased by $81 \%$ in individuals harboring SLCO3A1 rs8027174. Overall, AAG was the most relevant covariate based on the total reduction in $\mathrm{OFV}(\Delta \mathrm{OFV}=-45)$, although its inclusion only decreased unexplained residual variability by $7 \%$ and $10 \%$ for CL and $V$, respectively. Final model parameters are displayed in Table 3. Details of the covariate selection process can be found in the ESM. There was no apparent correlation between DRV AUC and viral load ( $W=582, p=0.45)$, CD4 lymphocyte count (rho $=0.09, p=0.3$ ), or occurrence of adverse events at any point during follow-up ( $W=1687$, $p=0.97$ for lipodystrophy; $W=1613.5, p=0.42$ for CNS adverse events).

\subsection{Goodness of Fit and Internal Validation}

There was good adequation between observed and individual predicted concentrations ( $r h o=0.87$ ), except for two outlier individuals with very high levels of AAG (2.66 and $2.2 \mathrm{~g} / \mathrm{L}$ ), thus low model-predicted CL, while their observed concentrations were actually low (see the ESM). A total of five further observations identified as outliers based on their weighted residuals were discarded, then the model was rerun. Figure 1 shows the goodness of fit after removal of these outliers. Normalized distribution prediction errors were normally distributed, with a mean of 0 and a variance of 1 (Fig. 2); $p$ values for the Shapiro-Wilk, Wilcoxon signedrank, and Fisher variance tests were 0.56, 0.84, and 0.79, respectively. Final parameter estimates were contained in the 95\% confidence intervals generated through nonparametric bootstrapping (Table 3 ).

\subsection{Target Attainment}

Table 4 shows the probability of target attainment (PTA) for each scenario, based on a representative population. Darunavir $C_{0}$ remained above the recommended cut-off of $0.055 \mathrm{mg} / \mathrm{L}$ in the majority of subjects for the $600-\mathrm{mg} \mathrm{q} 24 \mathrm{~h}$ and $400-\mathrm{mg}$ q $24 \mathrm{~h}$ strategies, while only $84.0 \%$ and $69.8 \%$ of individuals remained above $0.55 \mathrm{mg} / \mathrm{L}$ for these two respective strategies. The 2-mg/L target was reached in less than a third of patients, even with the standard once-daily dosage. Weekends-off SCT resulted in subtherapeutic concentrations 
Table 2 Genotypes

\begin{tabular}{|c|c|c|c|c|c|c|}
\hline Gene & rs & SNP & $n$ & $(\%)$ & $D$ & Notes \\
\hline \multirow[t]{5}{*}{$A B C B 1$} & rs2229109 & c. $1199 \mathrm{G}>\mathrm{A}$ & & & 0.13 & Missense variant \\
\hline & & $\mathrm{G} / \mathrm{G}$ & 116 & 91.3 & & \\
\hline & & $\mathrm{G} / \mathrm{A}$ & 8 & 6.3 & & \\
\hline & & A/A & 0 & 0 & & \\
\hline & & Missing & 3 & 2.4 & & \\
\hline \multirow[t]{5}{*}{$A B C B 1$} & rs 1045642 & c. $3435 \mathrm{C}>\mathrm{T}$ & & & 0.74 & Synonymous variant, decreases mRNA stability \\
\hline & & $\mathrm{C} / \mathrm{C}$ & 61 & 48.0 & & \\
\hline & & $\mathrm{C} / \mathrm{T}$ & 53 & 41.7 & & \\
\hline & & $\mathrm{T} / \mathrm{T}$ & 10 & 7.9 & & \\
\hline & & Missing & 3 & 2.4 & & \\
\hline \multirow[t]{5}{*}{ CYP3A5 } & rs776746 & g. $6986 \mathrm{~A}>\mathrm{G}$ & & & $-13.24 * *$ & $C Y P 3 A 5 * 3:$ truncated protein \\
\hline & & $* 1 / * 1$ & 33 & 26.0 & & \\
\hline & & $* 1 / * 3$ & 33 & 26.0 & & \\
\hline & & $* 3 / * 3$ & 58 & 45.7 & & \\
\hline & & Missing & 3 & 2.4 & & \\
\hline \multirow[t]{5}{*}{ СYР3A4 } & rs35599367 & g. $15389 \mathrm{C}>\mathrm{T}$ & & & 0.16 & CYP $3 A 4 * 22:$ alternative splice variant \\
\hline & & $* 1 / * 1$ & 115 & 90.6 & & \\
\hline & & $* 1 / * 22$ & 9 & 7.1 & & \\
\hline & & $* 22 / * 22$ & 0 & 0 & & \\
\hline & & Missing & 3 & 2.4 & & \\
\hline \multirow[t]{5}{*}{ SLCO3A1 } & rs8027174 & g. $91941607 \mathrm{G}>\mathrm{T}$ & & & 0.66 & Intron variant \\
\hline & & $\mathrm{G} / \mathrm{G}$ & 105 & 82.7 & & \\
\hline & & $\mathrm{G} / \mathrm{T}$ & 18 & 14.2 & & \\
\hline & & $\mathrm{T} / \mathrm{T}$ & 0 & 0 & & \\
\hline & & Missing & 4 & 3.1 & & \\
\hline \multirow[t]{5}{*}{ SLCO3A1 } & rs4294800 & g. $91917464 \mathrm{G}>\mathrm{A}$ & & & -1.11 & Intron variant \\
\hline & & $\mathrm{G} / \mathrm{G}$ & 56 & 44.1 & & \\
\hline & & G/A & 53 & 41.7 & & \\
\hline & & $\mathrm{A} / \mathrm{A}$ & 15 & 11.8 & & \\
\hline & & Missing & 3 & 2.4 & & \\
\hline
\end{tabular}

$D$ Haldane's D, $m R N A$ messenger RNA, SNP single nucleotide polymorphism

$* * p<0.05$ (statistically significant)

of DRV in a significant portion of individuals: $39.3 \%$ did not reach even the lowest $C_{0}$ target.

\section{Discussion}

Using population pharmacokinetics, we leveraged rich and sparse data to estimate individual PK parameters of DRV. A one-compartment model described the data well, with $\mathrm{CL}$ and $V$ being in the range of those previously estimated with one-compartment models in adult populations [22, 52]. The estimate of $k_{\mathrm{a}}$ was less precise, probably owing to a lack of data in the absorption phase, with its confidence interval encompassing lower values such as those found in Moltó et al. [19] and Dickinson et al. [53] all the way up to values closer to those calculated in Dickinson et al. [21] and Arab-Alameddine et al. [22]. Earlier publications have also applied two-compartment models [18, 19, 21, 53, 54] and more complex absorption kinetics such as transition compartment models [55], but these were not supported by our relatively sparse PK data. Among the investigated covariates, AAG and sex, along with $C Y P 3 A 5$ and SLCO3A1 genotypes, were found to be predictive of DRV pharmacokinetics.

Darunavir CL and $V$ both decreased with increasing AAG levels, which is consistent with previous reports of AAG affecting DRV pharmacokinetics [18, 19]. The clinical relevance of this association is unclear as total concentrations may be altered without any impact on unbound concentrations. In addition to the AAG level, the AAG phenotype is 
Table 3 Final population pharmacokinetic model

\begin{tabular}{lllll}
\hline & Estimate & RSE $(\%)$ & $95 \%$ CI & Shrinkage (\%) \\
\hline Structural model & & & & \\
$\mathrm{CL} / F\left(\mathrm{~L} \cdot \mathrm{h}^{-1}\right)$ & 12.9 & 5.3 & $11.3,14.4$ & 59 \\
$\omega_{\mathrm{CL}}(\mathrm{SD})$ & 0.22 & 13.95 & $0.08,0.28$ & \\
$V / F(\mathrm{~L})$ & 152 & 21.1 & 107,170 & 63 \\
$\omega_{V}(\mathrm{SD})$ & 0.33 & 16.36 & $0.05,0.39$ & \\
$k_{\mathrm{a}}\left(\mathrm{h}^{-1}\right)$ & 0.68 & 73.5 & $0.3,1.2$ & \\
$\omega_{\text {ka }}(\mathrm{SD})$ & 0.60 & 22.27 & $0.52,1.86$ & \\
Covariate model & & & & \\
AAG on CL & -0.61 & 37.9 & $-0.72,-0.43$ & \\
AAG on $V$ & -0.68 & 26.3 & $-0.74,-0.31$ & \\
Female sex on CL & -0.21 & 27.3 & $-0.31,-0.08$ & \\
$S L C O 3 A 1 G>T$ on $V$ & 0.81 & 59.5 & $0.34,2.23$ & \\
$C Y P 3 A 5 * 3$ on CL & -0.16 & 35.5 & $-0.28,-0.05$ & 12 \\
Residual variability & & & & \\
$\sigma_{\text {exponential }}(\mathrm{SD})$ & 0.281 & 27.7 & $0.15,0.32$ & \\
$\sigma_{\text {additive }}(\mathrm{SD})$ & 0.641 & 39.4 & $0.45,0.97$ & \\
\hline
\end{tabular}

$A A G \alpha 1$-acid glycoprotein, $C I$ confidence interval, $C L$ clearance, $C L / F$ apparent clearance, $k_{a}$ absorption rate constant, $R S E$ relative standard error (= standard error/parameter absolute value), $S D$ standard deviation, $V$ volume of distribution, $V / F$ apparent volume of distribution, $\omega$ random effect (inter-individual variability), $\sigma$ random effect (residual variability) known to modulate the plasma pharmacokinetics of some PIs, which may also play a role [56].

Regarding pharmacogenetics, both the loss-of-function variant $C Y P 3 A 5^{*} 3$ and Caucasian race were associated with smaller $V$ based on the rich dataset. However, the latter association was no longer apparent in the whole cohort and was not retained in the final model. $C Y P 3 A 5 * 3$ is commonly found in Caucasian individuals, which may result in CYP3A4 being the major isoform responsible for DRV metabolism for that ethnicity, while it is far less frequent in African individuals, in whom both isoforms (CYP3A4 and CYP3A5) may contribute to some extent. Indeed, in subjects receiving etravirine (a known CYP inducer), plasma exposure to DRV was previously found to be lower in carriers of the functional $C Y P 3 A 5 * 1$ allele compared with $C Y P 3 A 5 * 3$ carriers, suggesting that CYP3A5 is also involved in DRV biotransformation [57]. It is also worth noting that race correlated with the presence of the $C Y P 3 A 4 * 22, C Y P 3 A 5 * 3$, and $A B C B 13435 C>T$ alleles in this population. The fact that race remained a significant predictor in a model already including the $C Y P 3 A 5$ genotype suggests there may be other race-specific factors that we did not identify. Additionally, the SLCO3A1 rs8027174 variant was correlated with larger $V$ in our dataset. This intronic polymorphism was previously associated with smaller CL; however, it is unknown if DRV is a substrate of SLCO3A1 [19].

Lastly, DDIs were not identified as a significant covariate, most likely owing to the small number of clinically relevant interactions found in our patients (mostly limited to a few etravirine users) and because of RTV and COB boosting blunting any inductive effects or exceeding the inhibitory effects of concomitantly administered drugs.

Next, we used our model to perform simulations of alternative dosing schemes. There is growing interest in reducing the dose of DRV in virally suppressed patients: it was recently demonstrated that the daily dose of DRV/ RTV could be reduced from 800/100 to 400/100 mg q24h while maintaining adequate viral suppression in plasma and semen, although bioequivalence between the dosages could not be assessed [26, 54]. So far, it is unknown if a similar regimen could be used in patients using DRV/COB, especially as COB may not be quite as powerful as RTV. Moreover, dose reductions are typically performed in virally suppressed subjects. It would be important to assess their feasibility in more diverse groups of patients. With regard to ART simplification, one option would be "weekends-off" therapy (i.e., patients take ART for 5 days then interrupt for 2 days), although longer off-treatment phases may also be worth investigating.

In our simulations, for an unchanged COB dose, DRV $600 \mathrm{mg}$ q24h and $400 \mathrm{mg}$ q24h resulted in decreased exposure, but concentrations remained well above the target of $0.055 \mathrm{mg} / \mathrm{L}$ in the majority of subjects. Weekends-off therapy appeared unsafe from a purely PK point of view. Although concentrations fell below the cut-off of $0.55 \mathrm{mg} / \mathrm{L}$ in a significant portion of subjects, this value can be considered fairly conservative because it is already ten times higher than the typical wild-type $\mathrm{EC}_{50}$, and consequently does not apply to PI-naïve individuals. Finally, when targeting a $C_{0}$ of $2 \mathrm{mg} / \mathrm{L}$, the PTA was extremely low, even for the standard 

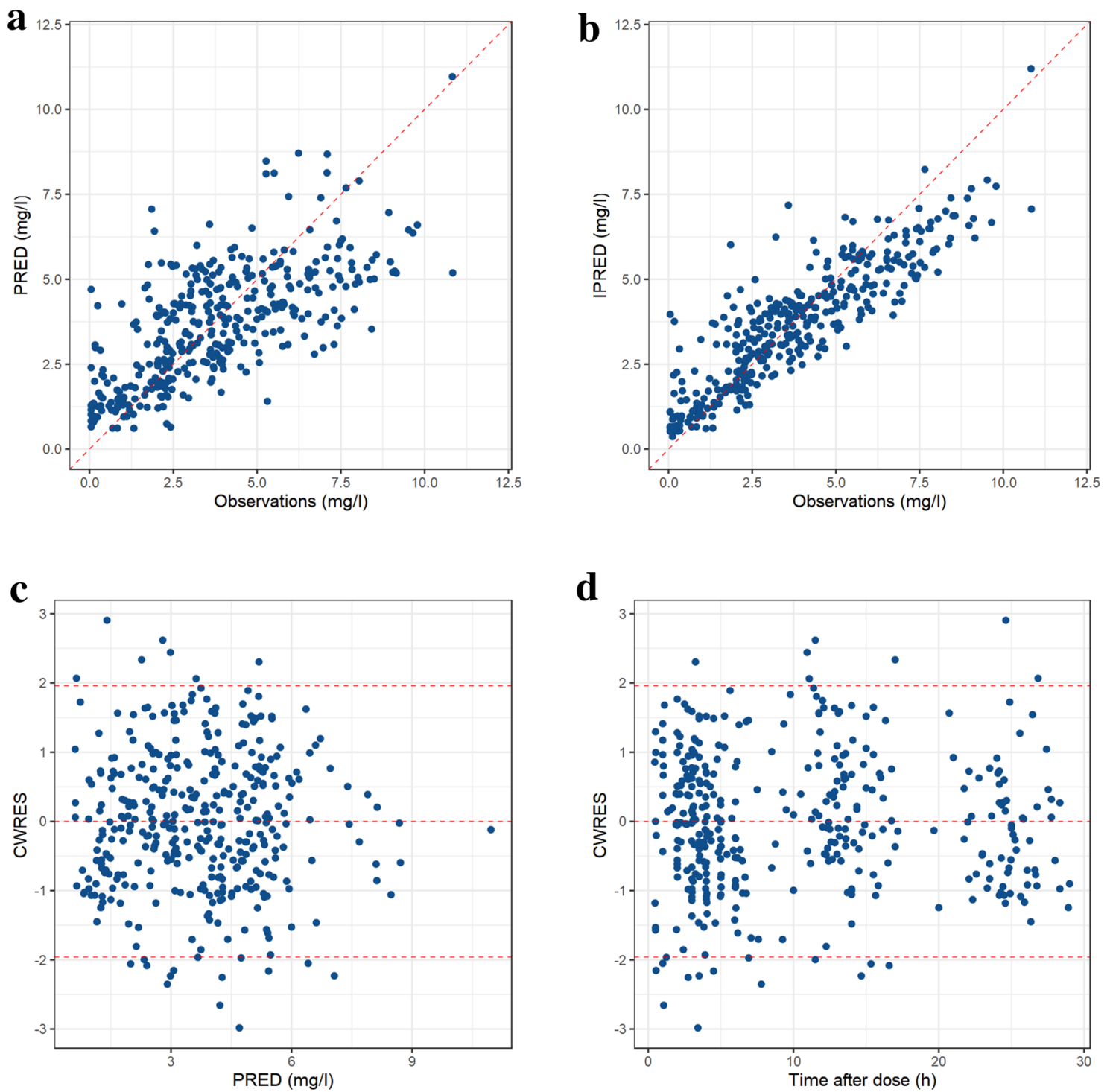

Fig. 1 Goodness of fit plots. a Population predicted concentrations (PRED) vs observations. b Individual predicted concentrations (IPRED) vs observations. c Conditional weighted residuals (CWRES) vs PRED. d CWRES vs time after dose

dosage. Interestingly, target achievement rates observed in our cohort were lower than the model-predicted rates: for q24h dosing, $7.1 \%$ and $25.7 \%$ of measured $C_{0}$ fell below $0.055 \mathrm{mg} / \mathrm{L}$ and below $0.55 \mathrm{mg} / \mathrm{L}$, respectively (compared with the model-predicted probabilities of $0.7 \%$ and $10.4 \%$ ). Among individuals with available $C_{0}$, only four had detectable viremia (comprised between 40 and 63 copies $/ \mathrm{mL}$ ), and only one individual had what could be considered as a low DRV $C_{0}(0.246 \mathrm{mg} / \mathrm{L})$. It is possible that in individuals with low DRV concentrations, concomitant ARVs ensured adequate anti-HIV activity, which would suggest that DRV is not a necessary component of the ARV regimen for those individuals, or it may be that the PK target itself is too stringent and that lower plasma concentrations are acceptable. Of course, plasma exposure is only a surrogate for intracellular accumulation and target-bound DRV. These results should be contrasted with those from a 4 days a week intermittent ART study, in which virological failure was rare despite low DRV exposure [37].

Additionally, the simulations used to derive PTA in the present paper were performed on a dataset that is representative of a certain patient population; it may not necessarily reflect what would be observed in populations with completely different genotypes, for instance. Further, PTA for groups such as children and pregnant women would have to be calculated based on data obtained in those groups specifically because of how much they differ from a standard adult population. For example, dosing recommendations for 

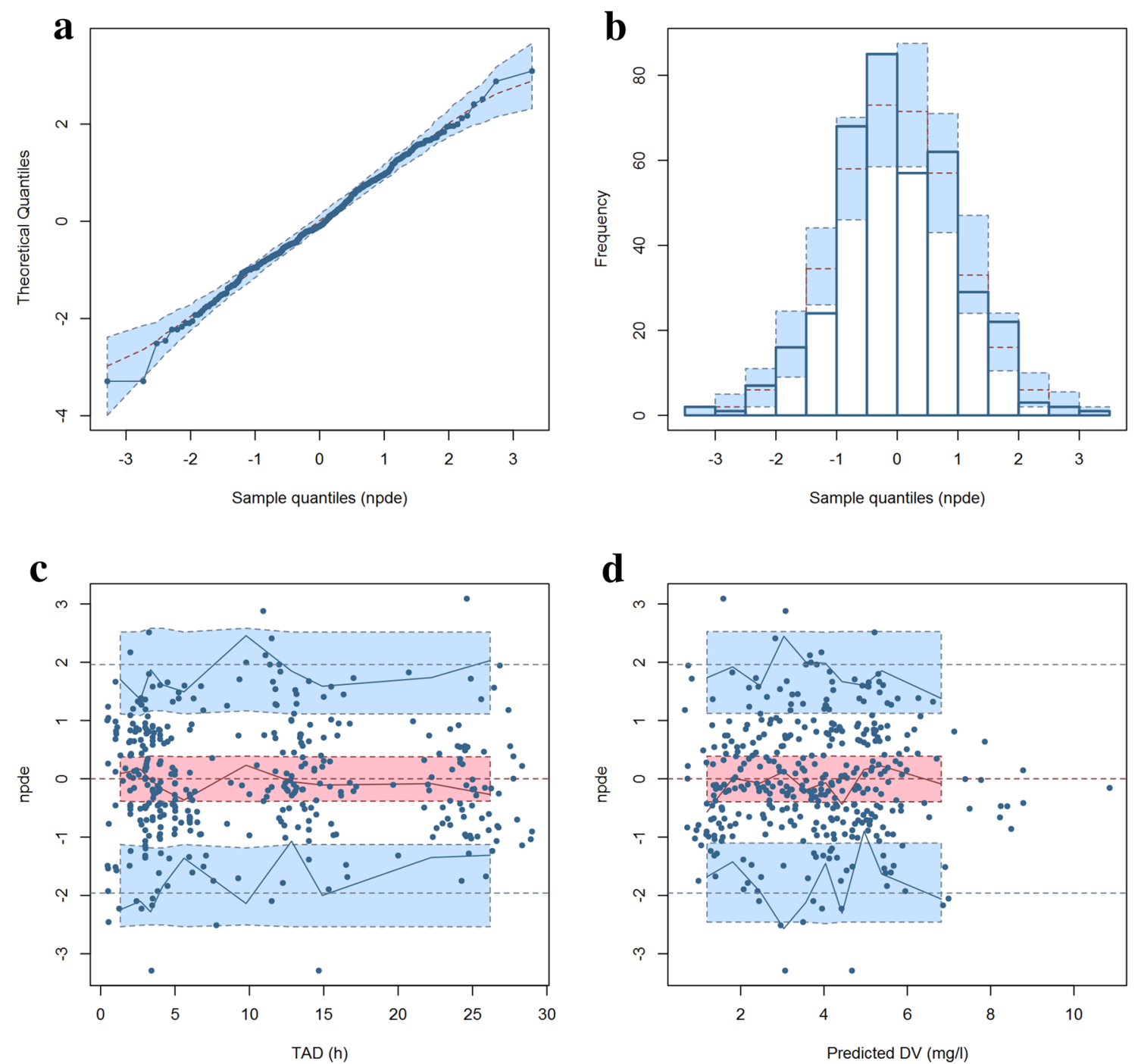

Fig. 2 Normalized distribution prediction error (NPDE). a $Q-Q$ plot of NPDE. b Histogram of NPDE. Shaded area represents theoretical distribution. c NPDE vs time after dose. Shaded areas represent the

prediction intervals associated with the 5th, 50th, and 95th percentiles. d NPDE vs predicted concentrations
Table 4 Probability of target attainment (PTA)

\begin{tabular}{lcccc}
\hline & $800 \mathrm{mg} \mathrm{q} 24 \mathrm{~h}$ & $600 \mathrm{mg} \mathrm{q} 24 \mathrm{~h}$ & $400 \mathrm{mg} \mathrm{q} 24 \mathrm{~h}$ & $\begin{array}{l}800 \mathrm{mg} \\
\mathrm{q} 24 \mathrm{~h} \\
5 / 7 \text { days }\end{array}$ \\
\hline$C_{0}(\mathrm{mg} / \mathrm{L})$ & & & 0.1 \\
$\mathrm{AUC}_{0-24}(\mathrm{mg} \cdot \mathrm{h} / \mathrm{L})$ & 76.2 & 1.17 & 38.1 & 6.5 \\
$\mathrm{PTA}(\%)$ & 57.2 & & \\
$C_{0}>0.055 \mathrm{mg} / \mathrm{L}$ & 99.3 & & 98.6 & 60.7 \\
$C_{0}>0.55 \mathrm{mg} / \mathrm{L}$ & 89.6 & 89.1 & 69.8 & 15.3 \\
$C_{0}>2 \mathrm{mg} / \mathrm{L}$ & 33.2 & 16.4 & 3.6 & 1.3 \\
\hline
\end{tabular}

$C_{0}$ and $\mathrm{AUC}_{0-24}$ are given as medians. For the $800 \mathrm{mg} 5 / 7$ days regimen, the $C_{0}$ is taken immediately before a new cycle (day 8 ) and the AUC is computed over day 7 to day 8

$A U C_{0-24}$ area under the curve from time 0 to $24 \mathrm{~h}, C_{0}$ trough concentration, $q 24 \mathrm{~h}$ every $24 \mathrm{~h}$ 
DRV/RTV in children aged 3-12 years have been derived using a PK model that incorporated not only AAG, but also body weight, as is often the case for pediatric models [58]. For pregnant women, in whom changes in CYP activity and protein binding can alter PK processes, a recent population PK study showed that administering DRV/RTV q12h instead of the standard q24h dosing could compensate for the reduction in exposure caused by pregnancy, thereby ensuring therapeutic drug concentrations, although systematic dose adjustments might not be warranted [23].

There were a few limitations to our study, the first of which was the sparseness of PK data in most subjects, for whom one sample per dose interval was obtained at a random post-intake time. This resulted in high levels of shrinkage, especially on $V$ and $\mathrm{k}_{\mathrm{a}}$, which could have prevented us from detecting all relevant covariate associations. Despite the addition of richer data and the use of a prior distribution, only a marginal improvement in model fit could be achieved and there was little change in individual parameter estimates. As a result, covariate associations should be regarded with caution. Further, there were few subjects using the $600 \mathrm{mg}$ $\mathrm{q} 12 \mathrm{~h}$ dosage in our cohort. The limited amount of information on this dose level could be problematic for simulations, as DRV exposure (namely, the AUC) is known to increase less than dose proportionally [23, 47]. Consequently, extrapolation to lower doses using simulations may yield more pessimistic results than expected (i.e., predicted AUC will be lower than its true value), although this would not change our general conclusions.

While the relationship between dose and total exposure is nonlinear, that between dose and unbound exposure may be linear [23], which brings us to another limitation of our study: only total concentrations were measured. With DRV being highly protein bound, it has been argued that unbound concentrations could be a useful biomarker for therapeutic drug monitoring (TDM) [59]. If DRV is assumed to have a low extraction ratio, displacement of its binding to AAG could affect the total concentration without influencing the unbound concentration, rendering the interpretation of TDM more delicate. Ultimately, as TDM for this drug traditionally relies on total concentrations and as PTA targets are defined based on protein binding-adjusted $\mathrm{EC}_{50}$, it was more rational to use the total concentration for the present work, but further studies are needed to establish the relationship between changes in total and unbound PK parameters, and how this could affect TDM of DRV. Lastly, most of the data used to build our model was collected in COB-boosted patients. While RTV and COB provide similar boosting effects, they are not identical and some minute differences have been demonstrated [16]; for instance, the boosting effect of COB was found to be insufficient in pregnant women [60]. Thus, model predictions could potentially be biased if applied to RTV-boosted patients.

\section{Conclusions}

Some individual factors were highlighted as potential predictors of DRV pharmacokinetics. Despite an important inter-individual variability, the probability of achieving commonly defined PK targets with reduced dose regimens was high, suggesting that this type of strategy could contribute to treatment simplification in a wide range of patients. Population PK models such as the one developed in this work could be used to predict changes in individual exposure based on a combination of TDM and covariate data.

Acknowledgements The authors thank all patients for their participation in the study. We are indebted to the clinical research nurses, Angeline Henry, Valérie Kin, and Françoise Leunen for their help in patient recruitment and sample collection. We also thank Kévin-Alexandre Delongie (Department of Clinical Chemistry, Cliniques Universitaires Saint-Luc) for technical assistance in drug quantification.

\section{Compliance with Ethical Standards}

Funding This work was supported by the Fonds pour la Formation à la Recherche dans l'Industrie et dans l'Agriculture (FRIA) [Grant number FC16749 to Gabriel Stillemans].

Conflict of interest Gabriel Stillemans, Leila Belkhir, Bernard Vandercam, Anne Vincent, Vincent Haufroid, and Laure Elens have no conflicts of interest that are directly relevant to the content of this article.

Ethics Approval The study protocol was approved by the local ethics committee (Comité d'Éthique Hospitalo-Facultaire des Cliniques Saint-Luc, approval number B403201731249). This study was conducted in accordance with the Declaration of Helsinki.

Consent to Participate Written informed consent was obtained from all participants before any study-related procedure.

Consent for Publication Not applicable.

Data Availability The datasets generated during and/or analyzed during the current study are available from the corresponding author on reasonable request.

Code Availability Not applicable.

Open Access This article is licensed under a Creative Commons Attribution-NonCommercial 4.0 International License, which permits any non-commercial use, sharing, adaptation, distribution and reproduction in any medium or format, as long as you give appropriate credit to the original author(s) and the source, provide a link to the Creative Commons licence, and indicate if changes were made. The images or other third party material in this article are included in the article's Creative Commons licence, unless indicated otherwise in a credit line to the material. If material is not included in the article's Creative Commons licence and your intended use is not permitted by statutory regulation or exceeds the permitted use, you will need to obtain permission directly from the copyright holder. To view a copy of this licence, visit http://creativecommons.org/licenses/by-nc/4.0/. 


\section{References}

1. European AIDS Clinical Society. Guidelines, version 10.0, November 2019. https://www.eacsociety.org/files/2019_guide lines-10.0_final.pdf. Accessed 02 Jul 2020.

2. Saag MS, Benson CA, Gandhi RT, Hoy JF, Landovitz RJ, Mugavero MJ, et al. Antiretroviral drugs for treatment and prevention of HIV infection in adults: 2018 recommendations of the International Antiviral Society-USA Panel. JAMA. 2018;320:379-96.

3. Ortiz R, DeJesus E, Khanlou H, Voronin E, van Lunzen J, Andrade-Villanueva J, et al. Efficacy and safety of once-daily darunavir/ritonavir versus lopinavir/ritonavir in treatment-naive HIV-1-infected patients at week 48. AIDS. 2008;22:1389-97.

4. Cahn P, Fourie J, Grinsztejn B, Hodder S, Molina J-M, Ruxrungtham $\mathrm{K}$, et al. Week 48 analysis of once-daily vs. twicedaily darunavir/ritonavir in treatment-experienced HIV-1-infected patients. AIDS. 2011;25:929-39.

5. Arasteh K, Yeni P, Pozniak A, Grinsztejn B, Jayaweera D, Roberts A, et al. Efficacy and safety of darunavir/ritonavir in treatmentexperienced HIV type-1 patients in the POWER 1, 2 and 3 trials at week 96. Antivir Ther. 2009;14:859.

6. Molina J-M, Cohen C, Katlama C, Grinsztejn B, Timerman A, de Pedro RJ, et al. Safety and efficacy of darunavir (TMC114) with low-dose ritonavir in treatment-experienced patients: 24-week results of POWER 3. J Acquir Immun Defic Syndr. 2007;46:24-31.

7. Haubrich R, Berger D, Chiliade P, Colson A, Conant M, Gallant J, et al. Week 24 efficacy and safety of TMC114/ritonavir in treatment-experienced HIV patients. AIDS. 2007;21:F11-8.

8. Madruga JV, Berger D, McMurchie M, Suter F, Banhegyi D, Ruxrungtham K, et al. Efficacy and safety of darunavir-ritonavir compared with that of lopinavir-ritonavir at 48 weeks in treatment-experienced, HIV-infected patients in TITAN: a randomised controlled phase III trial. Lancet. 2007;370:49-58.

9. Koh Y, Nakata H, Maeda K, Ogata H, Bilcer G, Devasamudram T, et al. Novel bis-tetrahydrofuranylurethane-containing nonpeptidic protease inhibitor (PI) UIC-94017 (TMC114) with potent activity against multi-PI-resistant human immunodeficiency virus in vitro. Antimicrob Agents Chemother. 2003;47:3123-9.

10. Dierynck I, De Wit M, Gustin E, Keuleers I, Vandersmissen J, Hallenberger S, et al. Binding kinetics of darunavir to human immunodeficiency virus type 1 protease explain the potent antiviral activity and high genetic barrier. J Virol. 2007;81:13845-51.

11. De Meyer S, Azijn H, Surleraux D, Jochmans D, Tahri A, Pauwels R, et al. TMC114, a novel human immunodeficiency virus type 1 protease inhibitor active against protease inhibitor-resistant viruses, including a broad range of clinical isolates. Antimicrob Agents Chemother. 2005;49:2314-21.

12. König SK, Herzog M, Theile D, Zembruski N, Haefeli WE, Weiss J. Impact of drug transporters on cellular resistance towards saquinavir and darunavir. J Antimicrob Chemother. 2010;65:2319-28.

13. Lepist E-I, Phan TK, Roy A, Tong L, MacLennan K, Murray B, et al. Cobicistat boosts the intestinal absorption of transport substrates, including HIV protease inhibitors and GS-7340, in vitro. Antimicrob Agents Chemother. 2012;56:5409-13.

14. Hartkoorn RC, Kwan WS, Shallcross V, Chaikan A, Liptrott $\mathrm{N}$, Egan D, et al. HIV protease inhibitors are substrates for OATP1A2, OATP1B1 and OATP1B3 and lopinavir plasma concentrations are influenced by SLCO1B1 polymorphisms. Pharmacogenet Genom. 2010;20:112-20.

15. Pharmaceutical and Medical Devices Agency. Darunavir/cobicistat. Module 2.4. Nonclinical overview. 2013. https://www. pmda.go.jp/drugs/2016/P20161125002/800155000_22800 AMX00714_F100_1.pdf. Accessed 02 Jul 2020.
16. Marzolini C, Gibbons S, Khoo S, Back D. Cobicistat versus ritonavir boosting and differences in the drug-drug interaction profiles with co-medications. J Antimicrob Chemother. 2016;71:1755-8.

17. Stillemans G, Belkhir L, Hesselink DA, Haufroid V, Elens L. Pharmacogenetic associations with cytochrome $\mathrm{P} 450$ in antiretroviral therapy: what does the future hold? Expert Opin Drug Metab Toxicol. 2018;14:601-11.

18. Vis P, Sekar V, van Schaick E, Hoetelmans R. Development and application of a population PK model of TMC114 in healthy volunteers and HIV-1-infected patients after administration of TMC114 in combination with low-dose ritonavir. PAGE 2006, Bruges, Belgium, 2006. https://www.page-meeting.org/defau 1t.asp?abstract $=964$. Accessed 02 Jul 2020.

19. Moltó J, Xinarianos G, Miranda C, Pushpakom S, Cedeño S, Clotet B, et al. Simultaneous pharmacogenetics-based population pharmacokinetic analysis of darunavir and ritonavir in HIVinfected patients. Clin Pharmacokinet. 2013;52:543-53.

20. Kakuda T, Sekar V, Vis P, Coate B, Ryan R, Anderson D, et al. Pharmacokinetics and pharmacodynamics of darunavir and etravirine in HIV-1-infected, treatment-experienced patients in the Gender, Race, and Clinical Experience (GRACE) Trial. AIDS Res Treat. 2012;2012:186987.

21. Dickinson L, Winston A, Boffito M, Khoo S, Back D, Siccardi M. Simulation of the impact of rifampicin on once-daily darunavir/ritonavir pharmacokinetics and dose adjustment strategies: a population pharmacokinetic approach. J Antimicrob Chemother. 2016;71:1041-5.

22. Arab-Alameddine M, Lubomirov R, Fayet-Mello A, Aouri M, Rotger M, Buclin T, et al. Population pharmacokinetic modelling and evaluation of different dosage regimens for darunavir and ritonavir in HIV-infected individuals. J Antimicrob Chemother. 2014;69:2489-98

23. Schalkwijk S, ter Heine R, Colbers A, Capparelli E, Best BM, Cressey TR, et al. Evaluating darunavir/ritonavir dosing regimens for HIV-positive pregnant women using semi-mechanistic pharmacokinetic modelling. J Antimicrob Chemother. 2019;74:1348-56.

24. Lanzafame M, Lattuada E, Rigo F, Ferrari A, Hill A, Vento S. Efficacy of a reduced dose of darunavir/ritonavir in a cohort of antiretroviral-naive and -experienced HIV-infected patients: a mediumterm follow-up. J Antimicrob Chemother. 2015;70:627-30.

25. Molto J, Valle M, Ferrer E, Domingo P, Curran A, Santos JR, et al. Reduced darunavir dose is as effective in maintaining HIV suppression as the standard dose in virologically suppressed HIVinfected patients: a randomized clinical trial. J Antimicrob Chemother. 2014;70:1139-45.

26. Molina J-M, Gallien S, Chaix M-L, El Abbassi EM, Madelaine I, Katlama C, et al. Low-dose ritonavir-boosted darunavir in virologically suppressed HIV-1-infected adults: an openlabel trial (ANRS 165 Darulight). J Antimicrob Chemother. 2018;73:2129-36.

27. Venter WDF, Moorhouse M, Sokhela S, Serenata C, Akpomiemie G, Qavi A, et al. Low-dose ritonavir-boosted darunavir once daily versus ritonavir-boosted lopinavir for participants with less than 50 HIV RNA copies per mL (WRHI 052): a randomised, openlabel, phase 3, non-inferiority trial. Lancet HIV. 2019;6:e428-37.

28. Lopez-Ruz MA, Navas P, López-Zúñiga MA, Gonzalvo MC, Sampedro A, Pasquau J, et al. Effect of monotherapy with darunavir/ritonavir on viral load in seminal fluid, and quality parameters of semen in HIV-1-positive patients. PLoS One. 2016;11:e0159305.

29. López-Ruz MA, López-Zúñiga MA, Gonzalvo MC, Sampedro A, Pasquau J, Hidalgo C, et al. Effect of monotherapy with darunavir/ cobicistat on viral load and semen quality of HIV-1 patients. PLoS One. 2018;13:e0196257. 
30. Gutierrez-Valencia A, Trujillo-Rodriguez M, Fernandez-Magdaleno T, Espinosa N, Viciana P, López-Cortés LF. Darunavir/ cobicistat showing similar effectiveness as darunavir/ritonavir monotherapy despite lower trough concentrations. J Int AIDS Soc. 2018;21:e25072.

31. Arribas J, Girard P-M, Paton N, Winston A, Marcelin A-G, Elbirt $\mathrm{D}$, et al. Efficacy of protease inhibitor monotherapy vs. triple therapy: meta-analysis of data from 2303 patients in 13 randomized trials. HIV Med. 2016;17:358-67.

32. Mena Á, Cid P, Dueñas C, Garcinuño MÁ, Lorenzo JF, Margusino L, et al. Darunavir/cobicistat maintains the effectiveness of darunavir/ritonavir in HIV-infected patients under mono or dual therapy. HIV Clin Trials. 2018;19:197-201.

33. Gianotti N, Galli L, Maserati R, Sighinolfi L, Ripamonti D, Palvarini $\mathrm{L}$, et al. Monotherapy with darunavir/ritonavir or lopinavir/ritonavir versus standard antiretroviral therapy: a randomized clinical trial (2 pm Study). New Microbiol. 2016;39:290-4.

34. Stöhr W, Dunn DT, Arenas-Pinto A, Orkin C, Clarke A, Williams I, et al. Factors associated with virological rebound in HIVinfected patients receiving protease inhibitor monotherapy. AIDS. 2016;30:2617-24.

35. Seang S, Schneider L, Nguyen T, Lê MP, Soulie C, Calin R, et al. Darunavir/ritonavir monotherapy at a low dose $(600 / 100 \mathrm{mg} /$ day $)$ in HIV-1-infected individuals with suppressed HIV viraemia. J Antimicrob Chemother. 2018;73:490-3.

36. Nachega JB, Parienti J-J, Uthman OA, Gross R, Dowdy DW, Sax $\mathrm{PE}$, et al. Lower pill burden and once-daily antiretroviral treatment regimens for HIV infection: a meta-analysis of randomized controlled trials. Clin Infect Dis. 2014;58:1297-307.

37. de Truchis P, Assoumou L, Landman R, Mathez D, Le Dû D, Bellet $\mathrm{J}$, et al. Four-days-a-week antiretroviral maintenance therapy in virologically controlled HIV-1-infected adults: the ANRS 162-4D trial. J Antimicrob Chemother. 2018;73:738-47.

38. Elens L, Veriter S, Di Fazio V, Vanbinst R, Boesmans D, Wallemacq P, et al. Quantification of 8 HIV-protease inhibitors and 2 nonnucleoside reverse transcriptase inhibitors by ultra-performance liquid chromatography with diode array detection. Clin Chem. 2008;55:170-4.

39. Charbe N, Baldelli S, Cozzi V, Castoldi S, Cattaneo D, Clementi E. Development of an HPLC-UV assay method for the simultaneous quantification of nine antiretroviral agents in the plasma of HIV-infected patients. J Pharm Anal. 2016;6:396-403.

40. Penchala SD, Fawcett S, Else L, Egan D, Amara A, Elliot E, et al. The development and application of a novel LC-MS/MS method for the measurement of dolutegravir, elvitegravir and cobicistat in human plasma. J Chromatogr B Anal Technol Biomed Life Sci. 2016;1027:174-80.

41. Graffelman J. Exploring diallelic genetic markers: the HardyWeinberg package. J Stat Softw. 2015;64:1-23.

42. Beal SL, Sheiner LB, Boeckmann A, Bauer R. NONMEM user's guides. Ellicott City: Icon Development Solutions; 2009.

43. Lindbom L, Ribbing J, Jonsson EN. Perl-speaks-NONMEM $(\mathrm{PsN})$ : a Perl module for NONMEM related programming. Comput Methods Progr Biomed. 2004;75:85-94.

44. R Core Team. R: a language and environment for statistical computing. Vienna, Austria: R Foundation for Statistical Computing; 2014. http://www.R-project.org/. Accessed 02 Jul 2020.

45. Comets E, Brendel K, Mentré F. Computing normalised prediction distribution errors to evaluate nonlinear mixed-effect models: the npde add-on package for R. Comput Methods Progr Biomed. 2008;90:154-66.

46. Gisleskog PO, Karlsson MO, Beal SL. Use of prior information to stabilize a population data analysis. J Pharmacokinet Pharmacodyn. 2002;29:473-505.

47. Sekar V, De Meyer S, Vangeneugden T, Lefebvre E, De Pauw $\mathrm{M}$, van Baelen B, et al. Pharmacokinetic/pharmacodynamic (PK/
PD) analyses of TMC114 in the POWER 1 and POWER 2 trials in treatment-experienced HIV-infected patients. In 13th Conference on retroviruses and opportunistic infections; Denver (CO); 5-8 February, 2006. http://www.natap.org/2006/CROI/CROI_37. htm. Accessed 02 Jul 2020.

48. US Food and Drug Administration. Drug development and drug interactions: table of substrates, inhibitors and inducers. 2020. https://www.fda.gov/drugs/drug-interactions-labeling/drug-devel opment-and-drug-interactions-table-substrates-inhibitors-andinducers. Accessed 02 Jul 2020.

49. Répertoire commenté des médicaments 2019. Centre belge d'information pharmacothérapeutique; 2019. https://www.cbip. be/ggr_pdfs/GGR_FR_2019.pdf. Accessed 02 Jul 2020.

50. Morlat P. Prise en charge médicale des personnes vivant avec le VIH: recommandations du groupe d'experts. Annexe pharmacologique. Conseil national du sida et des hépatites virales, Agence nationale de recherches sur le Sida et les hépatites virales; 2018. https://cns.sante.fr/actualites/prise-en-charge-du-vih-recom mandations-du-groupe-dexperts/. Accessed 02 Jul 2020.

51. Auton A, Abecasis GR, Altshuler DM, Durbin RM, Abecasis GR, Bentley DR, et al. A global reference for human genetic variation. Nature. 2015;526:68-74.

52. Daskapan A, Tran QTD, Cattaneo D, Gervasoni C, Resnati C, Stienstra Y, et al. Darunavir population pharmacokinetic model based on HIV outpatient data. Ther Drug Monit. 2019;41:59-65.

53. Dickinson L, Gurjar R, Stöhr W, Bonora S, Owen A, D'Avolio A, et al. Population pharmacokinetics and pharmacogenetics of ritonavir-boosted darunavir in the presence of raltegravir or tenofovir disoproxil fumarate/emtricitabine in HIV-infected adults and the relationship with virological response: a sub-study of the NEAT001/ANRS143 randomized trial. J Antimicrob Chemother. 2020;75:628-39.

54. Lê MP, Chaix M-L, Chevret S, Bertrand J, Raffi F, Gallien S, et al. Pharmacokinetic modelling of darunavir/ritonavir dose reduction (800/100 to $400 / 100 \mathrm{mg}$ once daily) in a darunavir/ritonavir-containing regimen in virologically suppressed HIV-infected patients: ANRS 165 DARULIGHT sub-study. J Antimicrob Chemother. 2018;73:2120-8.

55. Ter Heine R, Mulder JW, Van Gorp ECM, Wagenaar JFP, Beijnen $\mathrm{JH}$, Huitema ADR. Intracellular and plasma steady-state pharmacokinetics of raltegravir, darunavir, etravirine and ritonavir in heavily pre-treated HIV-infected patients: plasma and intracellular pharmacokinetics of new antiretroviral drugs. Br J Clin Pharmacol. 2010;69:475-83.

56. Colombo S, Buclin T, Decosterd L, Telenti A, Furrer H, Lee B, et al. Orosomucoid ( $\alpha 1$-acid glycoprotein) plasma concentration and genetic variants: effects on human immunodeficiency virus protease inhibitor clearance and cellular accumulation. Clin Pharmacol Ther. 2006;80:307-18.

57. Belkhir L, Elens L, Zech F, Panin N, Vincent A, Yombi JC, et al. Interaction between darunavir and etravirine is partly mediated by CYP3A5 polymorphism. PLoS One. 2016;11:e0165631.

58. Brochot A, Kakuda T, Van De Casteele T, Opsomer M, Tomaka F, Vermeulen A, et al. Model-based once-daily darunavir/ritonavir dosing recommendations in pediatric HIV-1-infected patients aged $\geq 3$ to $<12$ years. CPT Pharmacometr Syst Pharmacol. 2015;4:406-14.

59. Metsu D, Toutain PL, Chatelut E, Delobel P, Gandia P. Antiretroviral unbound concentration during pregnancy: piece of interest in the puzzle? J Antimicrob Chemother. 2017;72:2407-9.

60. Crauwels H, Osiyemi O, Zorrilla C, Bicer C, Brown K. Reduced exposure to darunavir and cobicistat in HIV-1-infected pregnant women receiving a darunavir/cobicistat-based regimen. HIV Med. 2019;20:337-43. 


\section{Affiliations}

\section{Gabriel Stillemans $^{1,2,5}\left(\right.$ Leila Belkhir $^{2,3}\left[\right.$ Bernard Vandercam $^{3} \cdot$ Anne Vincent $^{3} \cdot$ Vincent Haufroid $^{2,4}(\mathbb{D}$. Laure Elens ${ }^{1,2}$ (D)}

1 Integrated PharmacoMetrics, PharmacoGenomics and PharmacoKinetics, Louvain Drug Research Institute, Université Catholique de Louvain, Brussels, Belgium

2 Louvain Centre for Toxicology and Applied Pharmacology, Institut de Recherche Expérimentale et Clinique, Université Catholique de Louvain, Brussels, Belgium
3 AIDS Reference Center, Department of Internal Medicine, Cliniques Universitaires Saint-Luc, Université Catholique de Louvain, Brussels, Belgium

4 Department of Clinical Chemistry, Cliniques Universitaires Saint-Luc, Brussels, Belgium

5 Avenue E. Mounier 72, B01.72.02, Brussels, Belgium 